\title{
Attracting and Quasi-Invariant Sets of Cohen-Grossberg Neural Networks with Time Delay in the Leakage Term under Impulsive Perturbations
}

\author{
Guiying Chen ${ }^{1}$ and Linshan Wang ${ }^{2}$ \\ ${ }^{1}$ School of Mathematical Science, Liaocheng University, Liaocheng 252059, China \\ ${ }^{2}$ School of Mathematical Science, Ocean University of China, Qingdao 266100, China \\ Correspondence should be addressed to Linshan Wang; wangls@ouc.edu.cn
}

Received 24 September 2014; Accepted 27 December 2014

Academic Editor: Daoyi Xu

Copyright ( $) 2015$ G. Chen and L. Wang. This is an open access article distributed under the Creative Commons Attribution License, which permits unrestricted use, distribution, and reproduction in any medium, provided the original work is properly cited.

A class of impulsive Cohen-Grossberg neural networks with time delay in the leakage term is investigated. By using the method of $\mathscr{M}$-matrix and the technique of delay differential inequality, the attracting and invariant sets of the networks are obtained. The results in this paper extend and improve the earlier publications. An example is presented to illustrate the effectiveness of our conclusion.

\section{Introduction}

Cohen-Crossberg neural network model, which is initially proposed by Cohen and Grossberg [1] in 1983, has been found successful applications in many fields such as pattern recognition, parallel computing, associative memory, signal and image processing, and combinatorial optimization. Hence, there has been increasing interest in studying the stability and asymptotic behavior of this model with delays, impulses, and unique equilibrium, and many significant results have been obtained (see, e.g., [2-6]). However, the equilibrium point sometimes does not exist in many real physical systems, so it is an interesting subject to discuss the attracting and invariant sets of the neural networks $[7,8]$.

On the other hand, a leakage delay, which is the time delay in the leakage term and a factor affecting the stability of the system, has attracted considerable attentions (see, e.g., [913]). However, to the best of our knowledge, so far there are few results on the attracting and invariant sets of the CohenGrossberg neural networks with leakage delay. Motivated by the above discussion, in this paper, we investigate the attracting and quasi-invariant sets of a class of impulsive Cohen-Grossberg neural networks with leakage delay. By using the method of $\mathscr{M}$-matrix and the technique of delay differential inequality, the attracting and invariant sets of the addressed networks are obtained. The results in this paper extend and improve the earlier publications. An example is presented to illustrate the effectiveness of our conclusion.

\section{Model Description and Preliminaries}

Let $R^{n}$ be the space of $n$-dimensional real column vectors, $\mathcal{N} \triangleq\{1,2, \ldots, n\}, R_{+} \triangleq[0,+\infty), N \triangleq\{1,2, \ldots\}$, and $R^{m \times n}$ denotes the set of $m \times n$ real matrices. Usually, $E$ denotes an $n \times n$ unit matrix. For $A, B \in R^{m \times n}$ or $A, B \in R^{n}$, the notation $A \geq B(A \leq B, A>B, A<B)$ means that each pair of corresponding elements of $A$ and $B$ satisfies the inequality " $\geq(\leq,>,<)$ ". Particularly, $A$ is called a nonnegative matrix if $A \geq 0$, and $z \in R^{n}$ is called a positive vector if $z \geq 0$.

Let $\tau>0$; for $x(t)=\left(x_{1}(t), x_{2}(t), \ldots, x_{n}(t)\right)^{T}: R \rightarrow R^{n}$, we define

$$
\begin{gathered}
{[x(t)]^{+}=\left(\left|x_{1}(t)\right|,\left|x_{2}(t)\right|, \ldots,\left|x_{n}(t)\right|\right)^{T},} \\
{\left[x_{i}(t)\right]_{\tau}=\sup _{-\tau \leq s \leq 0}\left\{x_{i}(t+s)\right\},} \\
{[x(t)]_{\tau}=\left(\left[x_{1}(t)\right]_{\tau},\left[x_{2}(t)\right]_{\tau}, \ldots,\left[x_{n}(t)\right]_{\tau}\right)^{T},} \\
{[x(t)]_{\tau}^{+}=\left[[x(t)]^{+}\right]_{\tau} .}
\end{gathered}
$$


$C[X, Y]$ denotes the space of continuous mappings from the topological space $X$ to the topological space $Y$. Particularly, let $C \triangleq C\left[[-\tau, 0], R^{n}\right]$.

$P C^{1} \triangleq\left\{\phi:[-\tau, 0] \rightarrow R^{n}\right.$ is continuous and with continuous derivative everywhere except at finite number of point $t$ at which $\phi\left(t^{+}\right), \phi\left(t^{-}\right), \dot{\phi}\left(t^{+}\right)$, and $\dot{\phi}\left(t^{-}\right)$exist and $\phi\left(t^{+}\right)=$ $\phi(t), \dot{\phi}\left(t^{+}\right)=\dot{\phi}(t)$, where $\dot{\phi}$ denotes the derivative of $\left.\phi\right\} . P C^{1}$ is a space of piecewise right-hand continuous functions with the norm $\|\phi\|=\sup _{-\tau \leq s \leq 0}|\phi(s)|, \phi \in P C^{1}$, where $|\cdot|$ is a norm in $R^{n}$.

$P C\left[\left[t_{0}, \infty\right), R^{m \times n}\right] \triangleq\left\{\psi:\left[t_{0}, \infty\right) \rightarrow R^{m \times n} \mid \psi(t)\right.$ is continuous at $t \neq t_{k}, \psi\left(t_{k}^{+}\right)$and $\psi\left(t_{k}^{-}\right)$exist, $\psi\left(t_{k}\right)=\psi\left(t_{k}^{+}\right)$, for $k \in N\}$.

In this paper, we consider the following Cohen-Grossberg neural networks with impulses and time delays:

$$
\begin{gathered}
\dot{x}_{i}(t)=-\alpha_{i}(t, x(t))\left(\beta_{i}\left(x_{i}(t-\sigma)\right)-\sum_{j=1}^{n} a_{i j} f_{j}\left(x_{j}(t)\right)\right. \\
\left.-\sum_{j=1}^{n} b_{i j} g_{j}\left(x_{j}\left(t-\tau_{i j}(t)\right)\right)+I_{i}\right), \\
t \geq t_{0}, \quad t \neq t_{k}, \\
\Delta x_{i k}=x_{i}\left(t_{k}^{+}\right)-x_{i}\left(t_{k}^{-}\right) \\
=h_{i k}\left(x_{1}\left(t_{k}^{-}\right), x_{2}\left(t_{k}^{-}\right), \ldots, x_{n}\left(t_{k}^{-}\right)\right), \quad k \in N, \\
x_{i}\left(t_{0}+s\right)=\phi_{i}(s), \quad-\tau<s \leq 0, i=1,2, \ldots, n,
\end{gathered}
$$

where $n$ corresponds to the number of units in a neural network; $x_{i}(t)$ corresponds to the state of the $i$ th unit at time $t ; f_{j}$ and $g_{j}$ are the activation functions of the $j$ th unit; $\tau_{i j}(t)$ denotes the transmission delay and satisfies $0 \leq \tau_{i j}(t) \leq$ $r$ ( $r$ is a constant); $\sigma>0$ is the leakage delay. Consider $\tau=\max \{\sigma, r\} ; \alpha_{i}(t, x(t))>0$ represents the amplification function of the $i$ th neuron; $\beta_{i}\left(x_{i}(t)\right)$ is the behaved function at time $t$. Consider $(s)=\left(\phi_{1}(s), \ldots, \phi_{n}(s)\right)^{T} \in P C^{1}\left[[-\tau, 0], R^{n}\right]$. The fixed impulsive moments $t_{k}(k \in N)$ satisfy $\tau<t_{1}<t_{2}<$ $\cdots$ and $\lim _{k \rightarrow \infty} t_{k}=\infty$.

Definition 1 (see [14]). A function $x(t):\left[t_{0}-\tau, \infty\right) \rightarrow$ $R^{n}$ is said to be a solution of (2) through $\left(t_{0}, \phi\right)$, if $x(t) \in$ $P C\left[\left[t_{0}, \infty\right), R^{n}\right]$ as $t \geq t_{0}$, and satisfies (2) with the initial condition

$$
x\left(t_{0}+s\right)=\phi(s), \quad s \in[-\tau, 0], \phi \in P C^{1} .
$$

Throughout the paper, we always assume that, for any $\phi \in P C^{1}$, system (2) has at least one solution through $\left(t_{0}, \phi\right)$, denoted by $x\left(t, t_{0}, \phi\right)$ or $x_{t}\left(t_{0}, \phi\right)$ (simply $x(t)$ and $x_{t}$ if no confusion should occur), where $x_{t}\left(t_{0}, \phi\right)=x\left(t+s, t_{0}, \phi\right) \in$ $P C, s \in[-\tau, 0]$.

Definition 2 (see [7]). The set $S \subset P C^{1}$ is called a positive invariant set of (2), if for any initial value $\phi \in S$ we have the solution $x_{t}\left(t_{0}, \phi\right) \in S$ for $t \geq t_{0}$.
Definition 3 (see [7]). The set $S \subset P C^{1}$ is called a quasiinvariant set of (2), if there exist a matrix $W \geq 0$ and a vector $b \geq 0$ such that, for any $\phi \in S$, there exists a vector $z$ such that the solution $x(t)=x_{t}\left(t_{0}, \phi\right)$ of (2) satisfies $[x(t)]_{\tau}^{+} \leq W z+b$, $t \geq t_{0}$, as $[\phi]_{\tau}^{+} \leq z$. Obviously, the set $S$ is an invariant set of (2) if $W=E$ and $b=0$.

Definition 4 (see [7]). The set $S \subset P C^{1}$ is called a global attracting set of (2), if for any initial value $\phi \in P C^{1}$ the solution $x_{t}\left(t_{0}, \phi\right)$ converges to $S$ as $t \rightarrow+\infty$. That is,

$$
\operatorname{dist}\left(x_{t}, S\right) \longrightarrow 0, \quad t \longrightarrow+\infty,
$$

where $\operatorname{dist}(\varphi, S)=\inf _{\psi \in S} \operatorname{dist}(\varphi, \psi), \operatorname{dist}(\varphi, \psi)=$ $\sup _{s \in[-\tau, 0]}|\varphi(s)-\psi(s)|$ for $\varphi \in P C^{1}$.

Definition 5 (see [8]). The zero solution of (2) is said to be globally exponentially stable if for any solution $x\left(t, t_{0}, \phi\right)$ there exist constants $\lambda>0$ and $\kappa \geq 1$ such that $\left|x\left(t, t_{0}, \phi\right)\right| \leq$ $\kappa\|\phi\| e^{-\lambda\left(t-t_{0}\right)}, t \geq t_{0}$.

Definition 6 (see [15]). Let the matrix $D=\left(d_{i j}\right)_{n \times n}$ have nonpositive off-diagonal elements (i.e., $d_{i j} \leq 0, i \neq j$ ); then each of the following conditions is equivalent to the statement that $D$ is a nonsingular $\mathscr{M}$-matrix.

(i) All the leading principle minors of $D$ are positive.

(ii) $D=C-G$ and $\rho\left(C^{-1} G\right)<1$, where $G \geq 0$ and $C=$ $\operatorname{diag}\left\{c_{1}, \ldots, c_{n}\right\}$.

(iii) The diagonal elements of $D$ are all positive and there exists a positive vector $d$ such that $D d>0$ or $D^{T} d>0$.

For a nonsingular matrix $D \in R^{n \times n}$, we denote $\Omega_{M}(D) \triangleq$ $\left\{z \in R^{n}, z>0 \mid D z>0\right\}$.

Lemma 7 (see [14]). For a nonsingular $\mathscr{M}$-matrix $D, \Omega_{M}(D)$ is nonempty, and for any $z_{1}, z_{2} \in \Omega_{M}(D)$ we have

$$
k_{1} z_{1}+k_{2} z_{2} \in \Omega_{M}(D) \quad \forall k_{1}, k_{2}>0 .
$$

So $\Omega_{M}(D)$ is a cone without conical surface in $R^{n}$. We call it an "M-cone."

Lemma 8 (see [16]). Let $t_{0} \leq b \leq+\infty$ and $u(t) \in\left[\left[t_{0}, b\right), R^{n}\right]$ satisfy

$$
\begin{aligned}
D^{+}[u(t)]^{+} \leq R(t, u(t))\{ & P[u(t)]^{+} \\
& \left.+Q[u(t)]_{\tau}^{+}+\widehat{I}\right\}, \quad t \in\left[t_{0}, b\right), \\
u\left(t_{0}+s\right) \in & P C, \quad s \in[-\tau, 0],
\end{aligned}
$$

where $P=\left(p_{i j}\right)_{n \times n}, p_{i j} \geq 0,(i \neq j), Q=\left(q_{i j}\right)_{n \times n} \geq 0, \widehat{I}=$ $\left(\widehat{I}_{1}, \ldots, \widehat{I}_{n}\right)^{T} \geq 0, R(t, u)=\operatorname{diag}\left(R_{1}(t, u), \ldots, R_{n}(t, u)\right)$, and $R_{i}(t, u) \in C\left[\left[t_{0}, b\right) \times R_{n}, R_{+}\right], i \in \mathcal{N}$. Suppose that $-(P+Q)$ is a nonsingular $\mathscr{M}$-matrix.

(1) If the initial condition satisfies

$$
[u(t)]^{+} \leq x^{*}, \quad t_{0}-\tau \leq t \leq t_{0},
$$


where $x^{*}=\left(x_{1}^{*}, \ldots, x_{n}^{*}\right)^{T}=-d(P+Q)^{-1} \widehat{I}, d \geq 1$, then $[u(t)]^{+} \leq x^{*}$, for $t \geq t_{0}$.

(2) Suppose that there exist a scalar $\lambda>0$ and a vector $z=\left(z_{1}, \ldots, z_{n}\right)^{T}>0$ such that

$$
\left(\lambda E+P+Q e^{\lambda H \tau}\right) z \leq 0,
$$

where

$$
\begin{gathered}
H=\sup _{t \geq t_{0}} \max _{\left(s,[u]^{+}\right) \in[t-\tau, t] \times\left(0, x^{*}\right]} \widehat{R}(s, u(s))<\infty, \\
0 \leq \widehat{R}(t, u(t)) \leq \min _{1 \leq i \leq n}\left\{R_{i}(t, u(t))\right\} .
\end{gathered}
$$

If the initial condition satisfies

$$
[u(t)]^{+} \leq z e^{-\lambda \int_{t_{0}}^{t} \widehat{R}(s, u(s)) d s}-(P+Q)^{-1} \widehat{I}, \quad t_{0}-\tau \leq t \leq t_{0},
$$

then

$$
[u(t)]^{+} \leq z e^{-\lambda \int_{t_{0}}^{t} \widehat{R}(s, u(s)) d s}-(P+Q)^{-1} \widehat{I}, \quad t \geq t_{0} .
$$

\section{Main Results}

In this paper, we always suppose the following.

(A1) $\alpha_{i}(t, x(t)) \in C\left[\left[t_{0}, \infty\right) \times R^{n},\left[0, \bar{\alpha}_{i}\right]\right]$, where $\bar{\alpha}_{i}>0$ is a constant, $i \in \mathcal{N}$.

(A2) $\beta_{i}(\cdot)$ is differentiable, and there exist constants $\beta_{i}^{\prime}, \bar{\beta}_{i}>$ 0 such that $0<\beta_{i}^{\prime}<\dot{\beta}_{i}(t)<\bar{\beta}_{i}, i \in \mathcal{N}$, for any $t \in$ $\left[t_{0},+\infty\right)$.

(A3) $f_{i}(\cdot)$ and $g_{i}(\cdot)$ are Lipschitz continuous; that is, there exist constants $k_{i}$ and $l_{i}$ such that, for any $x_{1}, x_{2} \in R$,

$$
\begin{gathered}
\left|f_{i}\left(x_{1}\right)-f_{i}\left(x_{2}\right)\right| \leq k_{i}\left|x_{1}-x_{2}\right|, \\
\left|g_{i}\left(x_{1}\right)-g_{i}\left(x_{2}\right)\right| \leq l_{i}\left|x_{1}-x_{2}\right| .
\end{gathered}
$$

(A4) $-(\widehat{P}+\widehat{Q})$ is a nonsingular $\mathscr{M}$-matrix, where

$$
\begin{gathered}
\widehat{P}=\left(\widehat{p}_{i j}\right)_{n \times n}, \quad \widehat{Q}=\left(\widehat{q}_{i j}\right)_{n \times n}, \\
\widehat{p}_{i i}=-\beta_{i}^{\prime}+\left|a_{i i}\right| k_{i}, \quad \widehat{p}_{i j}=\left|a_{i j}\right| k_{j}(i \neq j), \\
\widehat{q}_{i i}=\sigma \bar{\alpha}_{i} \bar{\beta}_{i}^{2}+\left(1+\sigma \bar{\alpha}_{i} \bar{\beta}_{i}\right)\left|b_{i i}\right| l_{i}+\sigma \bar{\alpha}_{i} \bar{\beta}_{i}\left|a_{i i}\right| k_{i}, \\
\widehat{q}_{i j}=\left(1+\sigma \bar{\alpha}_{i} \bar{\beta}_{i}\right)\left|b_{i j}\right| l_{j}+\sigma \bar{\alpha}_{i} \bar{\beta}_{i}\left|a_{i j}\right| k_{j} .
\end{gathered}
$$

(A5) $\left[x+H_{k}(x)\right]^{+} \leq \Gamma_{k}[x]^{+}, k \in N$, for any $x \in R^{n}$, where $H_{k}(\cdot)=\left(h_{1 k}(\cdot), \ldots, h_{n k}(\cdot)\right)^{T}, \Gamma_{k}=\left(\gamma_{i j}^{(k)}\right)_{n \times n} \geq 0$.

(A6) For $z \in \Omega_{M}(-\widehat{P}-\widehat{Q})$,

$$
\begin{gathered}
\Gamma_{k} z \leq \mu_{k} z, \\
\Gamma_{k}(-\widehat{P}-\widehat{Q})^{-1} I^{*} \leq v_{k}(-\widehat{P}-\widehat{Q})^{-1} I^{*},
\end{gathered}
$$

where $I^{*}=\left(I_{1}^{*}, I_{1}^{*}, \ldots, I_{n}^{*}\right)^{T}, I_{i}^{*}=\left(1+\sigma \bar{\alpha}_{i} \bar{\beta}_{i}\right)\left(\left|\beta_{i}(0)\right|+\right.$ $\left.\sum_{j=1}^{n}\left(\left|a_{i j}\right|\left|f_{j}(0)\right|+\left|b_{i j}\right|\left|g_{j}(0)\right|\right)+\left|I_{i}\right|\right)+\sigma m_{i} \bar{\beta}_{i}$, and $\mu_{k}, v_{k} \geq 1$ satisfy

$$
\ln \mu_{k} \leq \lambda \int_{t_{k-1}}^{t_{k}} \widehat{\alpha}(s, x(s)) d s, \quad v=\sum_{k=1}^{n} \ln v_{k}<\infty, \quad k \in N,
$$

where $\widehat{\alpha}(s, x(s)) \triangleq \min _{1 \leq i \leq n}\left\{\alpha_{i}(s, x(s))\right\}$.

Theorem 9. Assume that (A1)-(A6) hold. Then, for any $\widehat{d} \geq 1$, the set $S_{\widehat{d}}=\left\{\phi \in P C^{1} \mid[\phi]_{\tau}^{+} \leq \widehat{d}(-\widehat{P}-\widehat{Q})^{-1} I^{*}\right\}$ is a quasiinvariant set of (2).

Proof. Combining with the middle value theorem, from (2) we can get

$$
\begin{aligned}
\frac{d x_{i}(t)}{d t}=-\alpha_{i}(t, x(t))( & \beta_{i}\left(x_{i}(t-\sigma)\right)-\sum_{j=1}^{n} a_{i j} f_{j}\left(x_{j}(t)\right) \\
& \left.-\sum_{j=1}^{n} b_{i j} g_{j}\left(x_{j}\left(t-\tau_{i j}(t)\right)\right)+I_{i}\right) \\
=\alpha_{i}(t, x(t))( & -\beta_{i}\left(x_{i}(t-\sigma)\right)+\beta_{i}\left(x_{i}(t)\right) \\
& -\beta_{i}\left(x_{i}(t)\right)+\sum_{j=1}^{n} a_{i j} f_{j}\left(x_{j}(t)\right) \\
& \left.+\sum_{j=1}^{n} b_{i j} g_{j}\left(x_{j}\left(t-\tau_{i j}(t)\right)\right)-I_{i}\right) \\
=\alpha_{i}(t, x(t))( & \dot{\beta}_{i}\left(x_{i}(t-(1-\theta) \sigma)\right) \\
& \times \dot{x}_{i}(t-(1-\theta) \sigma) \\
& -\beta_{i}\left(x_{i}(t)\right)+\sum_{j=1}^{n} a_{i j} f_{i}\left(x_{i}(t)\right) \\
& \left.+\sum_{j=1}^{n} b_{i j} g_{j}\left(x_{j}\left(t-\tau_{i j}(t)\right)\right)-I_{i}\right),
\end{aligned}
$$

where $0<\theta<1$.

Case 1. Let us first consider $t_{0} \leq t \leq t_{0}+(1-\theta) \sigma$. In this case $\dot{x}_{i}(t-(1-\theta) \sigma)=\dot{\phi}_{i}(t-(1-\theta) \sigma)=\dot{\phi}_{i}(s), s \in[-\tau, 0]$. Notice that $\phi \in P C^{1}$; there exist $m_{i}>0$ such that $\left[\dot{\phi}_{i}(s)\right]_{\tau} \leq m_{i}$, for all $i \in \mathcal{N}$. 
From (A1)-(A4) and (16), we have

$$
\begin{aligned}
& D^{+}\left|x_{i}(t)\right|=\operatorname{sgn}\left(x_{i}(t)\right) \frac{d x_{i}(t)}{d t} \\
& \leq \alpha_{i}(t, x(t))\left[-\beta_{i}^{\prime}\left|x_{i}(t)\right|+\sum_{j=1}^{n}\left|a_{i j}\right| k_{j}\left|x_{j}(t)\right|\right. \\
& +\sum_{j=1}^{n}\left|b_{i j}\right| l_{j}\left|x_{j}(t)\right|_{\tau} \\
& +\left(\left|\beta_{i}(0)\right|+\sum_{j=1}^{n}\left(\left|a_{i j}\right|\left|f_{j}(0)\right|\right.\right. \\
& \left.+\left|b_{i j}\right|\left|g_{j}(0)\right|\right) \\
& \left.+\left|I_{i}\right|+\sigma \bar{\beta} m_{i i}\right] \\
& \leq \alpha_{i}(t, x(t))\left(\sum_{j=1}^{n} \widehat{p}_{i j}\left|x_{j}(t)\right|\right. \\
& \left.+\sum_{j=1}^{n} \widehat{q}_{i j}\left|x_{j}(t)\right|_{\tau+(1-\theta) \sigma}+I_{i}^{*}\right) .
\end{aligned}
$$

Hence,

$$
\begin{array}{r}
D^{+}[x(t)]^{+} \leq \alpha(t, x(t))\left(\widehat{P}[x(t)]^{+}+\widehat{Q}[x(t)]_{\tau+(1-\theta) \sigma}^{+}+I^{*}\right), \\
t_{0} \leq t \leq t_{0}+(1-\theta) \sigma .
\end{array}
$$

Case 2. Let us consider $t \geq t_{0}+(1-\theta) \sigma$. From (16), we get

$$
\begin{aligned}
& \frac{d x_{i}(t)}{d t}=-\alpha_{i}(t, x(t)) \\
& \times\left\{\sigma \dot { \beta } _ { i } \left(x_{i}(t-(1-\theta) \sigma)\right.\right. \\
& \times\left[-\alpha_{i}\left(x_{i}(t-(1-\theta) \sigma)\right)\right. \\
& \times\left(\beta_{i}\left(x_{i}(t-(2-\theta) \sigma)\right)\right) \\
& -\sum_{j=1}^{n} a_{i j} f_{j}\left(x_{j}(t-(1-\theta) \sigma)\right) \\
& -\sum_{j=1}^{n} b_{i j} g_{j}\left(x_{j}(t-(1-\theta) \sigma)\right)
\end{aligned}
$$

$$
\left.\begin{array}{r}
\left.\left.\left.-\tau_{i j}(t-(1-\theta) \sigma)\right)+I_{i}\right)\right] \\
-\beta_{i}\left(x_{i}(t)\right)+\sum_{j=1}^{n} a_{i j} f_{j}\left(x_{j}(t)\right) \\
+\sum_{j=1}^{n} b_{i j} g_{j}\left(x_{j}\left(t-\tau_{i j}(t)\right)\right)-I_{i}
\end{array}\right\} .
$$

Then from (A1)-(A4), we have

$$
\begin{aligned}
D^{+}\left|x_{i}(t)\right|= & \operatorname{sgn}\left(x_{i}(t)\right) \frac{d x_{i}(t)}{d t} \\
\leq & \alpha_{i}(t, x(t)) \\
& \times\left\{\sigma \overline { \beta } _ { i } \left[\overline { \alpha } _ { i } \left(\bar{\beta}_{i}\left|x_{i}(t)\right|_{\tau+(1-\theta) \sigma}+\left|\beta_{i}(0)\right|\right.\right.\right. \\
+ & \sum_{j=1}^{n}\left|a_{i j}\right|\left(k_{j}\left|x_{j}(t)\right|_{\tau+(1-\theta) \sigma}\right. \\
+ & \left.+\sum_{j=1}^{n}\left|b_{i j}\right|(0) \mid\right) \\
+\left.\left|l_{j}\right| x_{j}(t)\right|_{\tau+(1-\theta) \sigma} & \left.+\left|g_{j}(0)\right|\right)
\end{aligned}
$$$$
-\beta_{i}^{\prime}\left|x_{i}(t)\right|+\left|\beta_{i}(0)\right|
$$$$
+\sum_{j=1}^{n}\left|a_{i j}\right|\left(k_{j}\left|x_{j}(t)\right|+\left|f_{j}(0)\right|\right)
$$$$
+\sum_{j=1}^{n}\left|b_{i j}\right| l_{j} \mid\left(\left.x_{j}(t)\right|_{\tau+(1-\theta) \sigma}\right.
$$$$
\left.\left.+\left|g_{j}(0)\right|\right)+\left|I_{i}^{*}\right|\right\}
$$$$
=\alpha_{i}(t, x(t))\left(\sum_{j=1}^{n} \widehat{p}_{i j}\left|x_{j}(t)\right|\right.
$$

$$
\left.+\sum_{j=1}^{n} \widehat{q}_{i j}\left|x_{j}(t)\right|_{\tau+(1-\theta) \sigma}+I_{i}^{*}\right) .
$$


From (17) and (20), we get

$$
\begin{array}{r}
D^{+}[x(t)]^{+} \leq \alpha(t, x(t))\left(\widehat{P}[x(t)]^{+}+\widehat{Q}[x(t)]_{\tau+(1-\theta) \sigma}^{+}+I^{*}\right), \\
t \in\left[t_{k}, t_{k+1}\right), \quad k \in N .
\end{array}
$$

For the initial conditions $x\left(t_{0}+s\right)=\phi(s), s \in[-\tau, 0]$, where $\phi(s) \in S_{\widehat{d}}$, we have

$$
[x(t)]^{+} \leq-\widehat{d}(\widehat{P}+\widehat{Q})^{-1} I^{*}, \quad t_{0}-\tau \leq t \leq t_{0} .
$$

By (21) and Lemma 8, we have

$$
[x(t)]^{+} \leq-\widehat{d}(\widehat{P}+\widehat{Q})^{-1} I^{*}, \quad t_{0} \leq t \leq t_{1} .
$$

Suppose that for all $m=1,2, \ldots, k$ the inequalities

$$
[x(t)]^{+} \leq-v_{0} \ldots v_{m-1} \widehat{d}(\widehat{P}+\widehat{Q})^{-1} I^{*}, \quad t_{m-1} \leq t<t_{m},
$$

hold, where $v_{0}=1$. Then, from (A5) and (A6),

$$
\begin{aligned}
{\left[x\left(t_{k}\right)\right]^{+} } & =\left[x\left(t_{k}^{-}\right)+H_{k}\left(x\left(t_{k}^{-}\right)\right)\right]^{+} \leq \Gamma_{k}\left[x\left(t_{k}^{-}\right)\right]^{+} \\
& \leq \Gamma_{k}\left(v_{0} \cdots v_{k-1} \widehat{d}(-\widehat{P}-\widehat{Q})^{-1} I^{*}\right) \\
& \leq v_{0} \cdots v_{k} \widehat{d}(-\widehat{P}-\widehat{Q})^{-1} I^{*} .
\end{aligned}
$$

This, together with $v_{k} \geq 1$, leads to

$$
[x(t)]^{+} \leq v_{0} \cdots v_{k} \widehat{d}(-\widehat{P}-\widehat{Q})^{-1} I^{*}, \quad \text { for } t \in\left[t_{k}-\tau, t_{k}\right] .
$$

On the other hand,

$$
\begin{aligned}
D^{+}[x(t)]^{+} \leq \alpha(t, x(t))\left(\widehat{P}[x(t)]^{+}+\widehat{Q}[x(t)]_{\tau+(1-\theta) \sigma}^{+}\right. \\
\left.+v_{0} \cdots v_{k} I^{*}\right), \quad t \neq t_{k} .
\end{aligned}
$$

It follows from (A4), (26), (27), and Lemma 8 that

$$
[x(t)]^{+} \leq v_{0} \cdots v_{k} \widehat{d}(-\widehat{P}-\widehat{Q})^{-1} I^{*} \quad \text { for } t \in\left[t_{k}, t_{k+1}\right) .
$$

By the induction, we can conclude that

$$
[x(t)]^{+} \leq v_{0} \cdots v_{k} \widehat{d}(-\widehat{P}-\widehat{Q})^{-1} I^{*}, \quad t \in\left[t_{k-1}, t_{k}\right), k \in N .
$$

From (A6),

$$
v_{0} \cdots v_{k} \leq e^{v}
$$

and we have

$$
[x(t)]^{+} \leq e^{v} \widehat{d}(-\widehat{P}-\widehat{Q})^{-1} I^{*}, \quad t \in\left[t_{0}, t_{k}\right), k \in N .
$$

This implies that the conclusion holds and the proof is complete.
Theorem 10. Assume that (A1)-(A6) hold. Then the set $S=$ $\left\{\phi \in P C^{1} \mid[\phi]_{\tau}^{+} \leq e^{v}(-\widehat{P}-\widehat{Q})^{-1} I^{*}\right\}$ is a global attracting set of (2).

Proof. By a similar proof of Theorem 9, we can get (21) and (31).

By continuity, we can find $\lambda>0$ and an enough small $\varepsilon>0$ such that

$$
\left((\lambda+\varepsilon) E+\widehat{P}+\widehat{Q} e^{\lambda H(\tau+\sigma)}\right) z<0
$$

where

$$
\begin{gathered}
H \triangleq \sup _{t \geq t_{0}} \max _{s \in[t-\tau, t], x \in\left[0, x^{*}\right]} \widehat{\alpha}(s, x(s))<\infty, \\
\widehat{\alpha}(s, x(s)) \triangleq \min _{1 \leq i \leq n}\left\{\alpha_{i}(s, x(s))\right\}, \\
x^{*}=-e^{2 v}(-\widehat{P}-\widehat{Q})^{-1} I^{*} .
\end{gathered}
$$

For the initial conditions $x\left(t_{0}+s\right)=\phi(s), s \in[-\tau, 0]$, where $\phi(s) \in P C^{1}$, we have

$$
[x(t)]^{+} \leq \kappa_{0} z, \quad \kappa_{0}=\frac{\|\phi\|}{\min _{1 \leq i \leq n}\left\{z_{i}\right\}}, t_{0}-\tau \leq t \leq t_{0},
$$

and so

$$
\begin{array}{r}
{[x(t)]^{+} \leq \kappa_{0} z e^{-(\lambda+\varepsilon) \int_{t_{0}}^{t} \widehat{\alpha}(s, x(s)) d s}-(\widehat{P}+\widehat{Q})^{-1} I^{*},} \\
t_{0}-\tau \leq t \leq t_{0} .
\end{array}
$$

By the induction and Lemma 8 , we can conclude that

$$
\begin{aligned}
{[x(t)]^{+} \leq } & \mu_{0} \cdots \mu_{k} \kappa_{0} z e^{-(\lambda+\varepsilon) \int_{t_{0}}^{t} \widehat{\alpha}(s, x(s)) d s} \\
& +v_{0} \cdots v_{k}(-\widehat{P}-\widehat{Q})^{-1} I^{*}, \quad t \in\left[t_{k-1}, t_{k}\right), k \in N .
\end{aligned}
$$

From (A6), we conclude that

$$
\begin{aligned}
{[x(t)]^{+} \leq } & e^{\lambda\left(t_{1}-t_{0}\right)} \cdots e^{\lambda\left(t_{k-1}-t_{k-2}\right)} \kappa_{0} z e^{-(\lambda+\varepsilon) \int_{t_{0}}^{t} \widehat{\alpha}(s, x(s)) d s} \\
& +v_{0} \cdots v_{k}(-\widehat{P}-\widehat{Q})^{-1} I^{*} \\
\leq & \kappa_{0} z e^{-\varepsilon \int_{t_{0}}^{t} \widehat{\alpha}(s, x(s)) d s} \\
& +e^{v}(-\widehat{P}-\widehat{Q})^{-1} I^{*}, \quad t \in\left[t_{0}, t_{k}\right), k \in N .
\end{aligned}
$$

This implies that the conclusion holds and the proof is complete.

Theorem 11. Assume that (A1)-(A5) with $\Gamma_{k}=E$ hold. Then $S=\left\{\phi \in P C^{1} \mid[\phi]_{\tau}^{+} \leq(-\widehat{P}-\widehat{Q})^{-1} I^{*}\right\}$ is a positive invariant set and also a global attracting set of (2).

Proof (straightforward). Obviously, if $\alpha(0)=0$, then $x(t)=0$ is a solution of (2). 
Corollary 12. Assume that (A1)-(A5) with $\alpha_{i}(t, x(t)) \geq \delta>$ $0, I^{*}=0$ hold. If

$$
\ln \mu_{k} \leq \lambda\left(t_{k}-t_{k-1}\right),
$$

where $\mu_{k} \geq 1$ satisfy $\Gamma_{k} z \leq \mu_{k} z, k \in N$, then the zero solution of (2) is globally exponentially stable.

Remark 13. If $\sigma=0$, and $H_{k}(\cdot) \equiv 0$, then from Theorems 9 and 10 we can get Theorem 5.1 and Corollary 5.1 in [16].

Remark 14. If $\sigma=0$, and $H_{k}(\cdot) \equiv 0$, then from Corollary 12 we can get Corollary 3.1 in [5].

\section{Illustrative Example}

Consider the Cohen-Grossberg neural networks (2) with the following parameters, activation functions, amplification functions, behaved functions, and delay functions ( $n=$ $2, i, j=1,2)$ :

$$
\begin{gathered}
a_{11}=0.2, \quad a_{12}=0.1, \quad a_{21}=0.2, \quad a_{22}=0.3, \\
b_{11}=0.2, \quad b_{12}=0.3, \quad b_{21}=0.2, \quad b_{22}=0.5, \\
J_{1}=\frac{1}{2}=J_{2}, \quad \sigma=0.2, \\
\alpha_{1}(t, x(t))=\max \left\{\frac{1}{2},\left|\sin \left(x_{1}(t)\right)\right|\right\} \\
\alpha_{2}(t, x(t))=\max \left\{\frac{1}{2},\left|\sin \left(2 x_{2}(t)\right)\right|\right\} \\
\beta_{1}\left(x_{1}(t)\right)=3 x_{1}(t), \quad \beta_{2}\left(x_{2}(t)\right)=2 x_{2}(t), \\
f_{1}\left(x_{1}\right)=g_{1}\left(x_{1}\right)=\frac{1}{2}\left(\left|x_{1}+1\right|-\left|x_{1}-1\right|\right) \\
f_{2}\left(x_{2}\right)=g_{2}\left(x_{2}\right)=x_{2}, \tau_{i j}(t)=|\sin (j t)| \\
\Gamma_{k}=e^{1 / 2^{k}} E .
\end{gathered}
$$

Obviously, $k=l=1, H=1 / 2, \mu_{k}=v_{k}=e^{1 / 2^{k}}$, and $v=1$. The parameters of condition (A4) are as follows:

$$
\begin{gathered}
\widehat{P}=\left(\begin{array}{cc}
-2.8 & 0.1 \\
0.2 & -1.7
\end{array}\right), \quad \widehat{Q}=\left(\begin{array}{ll}
2.04 & 0.24 \\
0.16 & 1.12
\end{array}\right), \\
-(\widehat{P}+\widehat{Q})=\left(\begin{array}{cc}
0.76 & -0.34 \\
-0.36 & 0.58
\end{array}\right) .
\end{gathered}
$$

We can easily observe that $-(\widehat{P}+\widehat{Q})$ is a nonsingular $\mathscr{M}$ matrix and

$$
\Omega_{M}(-\widehat{P}-\widehat{Q})=\left\{\left(z_{1}, z_{2}\right)^{T}>0 \mid \frac{18}{29} z_{1}<z_{2}<\frac{38}{17} z_{1}\right\} .
$$

Let $z=(1,1)^{T} \in \Omega_{M}(-\widehat{P}-\widehat{Q})$ and $\lambda=0.082$ which satisfies the inequality

$$
\left(\lambda E+\widehat{P}+\widehat{Q} e^{\lambda H(\tau+\sigma)}\right) z=(-0.223,-1.123)^{T}<0 .
$$

Clearly, all conditions of Theorems 9 and 10 are satisfied. So $S=\left\{\phi \in P C^{1} \mid[\phi]_{\tau}^{+} \leq(-\widehat{P}-\widehat{Q})^{-1} I^{*}\right\}=(31.89,32.92)^{T}$ is a quasi-invariant set and also a global attracting set of (2).

\section{Conflict of Interests}

The authors declare that there is no conflict of interests regarding the publication of this paper.

\section{Acknowledgments}

This work was supported by the National Natural Science Foundation of China (11171374) and Natural Science Foundation of Shandong Province (ZR2011AZ001).

\section{References}

[1] M. A. Cohen and S. Grossberg, "Absolute stability of global pattern formation and parallel memory storage by competitive neural networks," IEEE Transactions on Systems, Man, and Cybernetics, vol. 13, no. 5, pp. 815-826, 1983.

[2] H. Lu, R. Shen, and F.-L. Chung, "Global exponential convergence of Cohen-Grossberg neural networks with time delays," IEEE Transactions on Neural Networks, vol. 16, no. 6, pp. 16941696, 2005.

[3] S. Guo and L. Huang, "Stability analysis of Cohen-Grossberg neural networks," IEEE Transactions on Neural Networks, vol. 17, no. 1, pp. 106-117, 2006.

[4] Y. Chen, "Global asymptotic stability of delayed CohenGrossberg neural networks," IEEE Transactions on Circuits and Systems I: Regular Papers, vol. 53, no. 2, pp. 351-357, 2006.

[5] Z. Yang and D. Xu, "Impulsive effects on stability of CohenGrossberg neural networks with variable delays," Applied Mathematics and Computation, vol. 177, no. 1, pp. 63-78, 2006.

[6] O. Faydasicok and S. Arik, "Robust stability analysis of a class of neural networks with discrete time delays," Neural Networks, vol. 29-30, pp. 52-59, 2012.

[7] D. Xu and Z. Yang, "Attracting and invariant sets for a class of impulsive functional differential equations," Journal of Mathematical Analysis and Applications, vol. 329, no. 2, pp. 1036-1044, 2007.

[8] D. Y. Xu and S. J. Long, "Attracting and quasi-invariant sets of non-autonomous neural networks with delays," Neurocomputing, vol. 77, no. 1, pp. 222-228, 2012.

[9] K. Gopalsamy, Stability and Oscillations in Delay Differential Equations of Population Dynamics, vol. 74 of Mathematics and its Applications, Kluwer Academic Publishers, Dordrecht, Netherlands, 1992.

[10] K. Gopalsamy, "Leakage delays in BAM," Journal of Mathematical Analysis and Applications, vol. 325, no. 2, pp. 1117-1132, 2007.

[11] X. Li, X. Fu, P. Balasubramaniam, and R. Rakkiyappan, "Existence, uniqueness and stability analysis of recurrent neural networks with time delay in the leakage term under impulsive perturbations," Nonlinear Analysis. Real World Applications, vol. 11, no. 5, pp. 4092-4108, 2010.

[12] M. J. Park, O. M. Kwon, J. H. Park, S. M. Lee, and E. J. Cha, "Synchronization criteria for coupled stochastic neural networks with time-varying delays and leakage delay," Journal of the Franklin Institute, vol. 349, no. 5, pp. 1699-1720, 2012. 
[13] G. Y. Chen and L. S. Wang, "Global exponential Robust stability of static interval neural networks with time delay in the leakage term," Journal of Applied Mathematics, vol. 2014, Article ID 972608, 6 pages, 2014.

[14] D. Xu and Z. Yang, "Impulsive delay differential inequality and stability of neural networks," Journal of Mathematical Analysis and Applications, vol. 305, no. 1, pp. 107-120, 2005.

[15] A. Berman and R. J. Plemmons, Nonnegative Matrices in the Mathematical Sciences, Academic Press, New York, NY, USA, 1979.

[16] D. $\mathrm{Xu}$ and L. $\mathrm{Xu}$, "New results for studying a certain class of nonlinear delay differential systems," IEEE Transactions on Automatic Control, vol. 55, no. 7, pp. 1641-1645, 2010. 


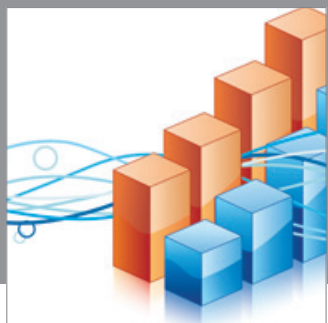

Advances in

Operations Research

mansans

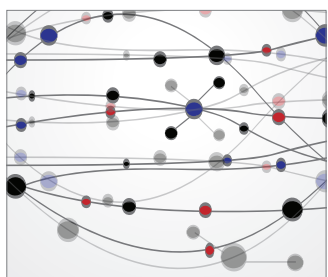

The Scientific World Journal
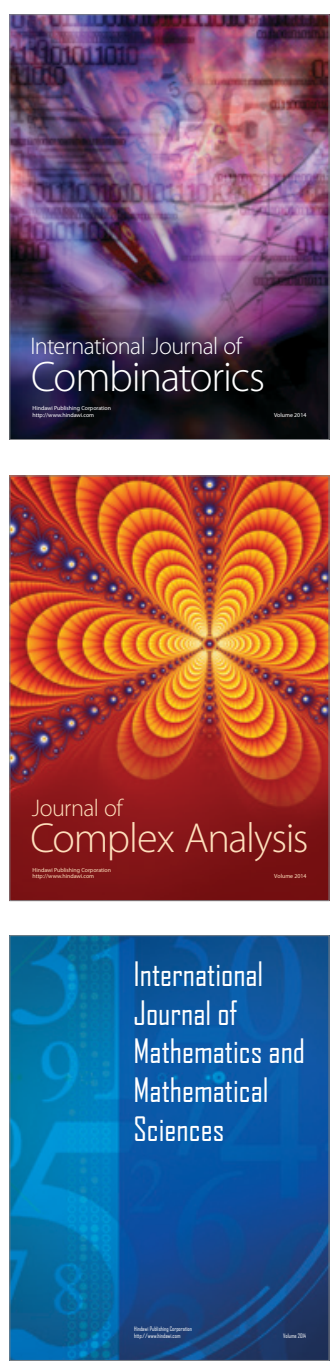
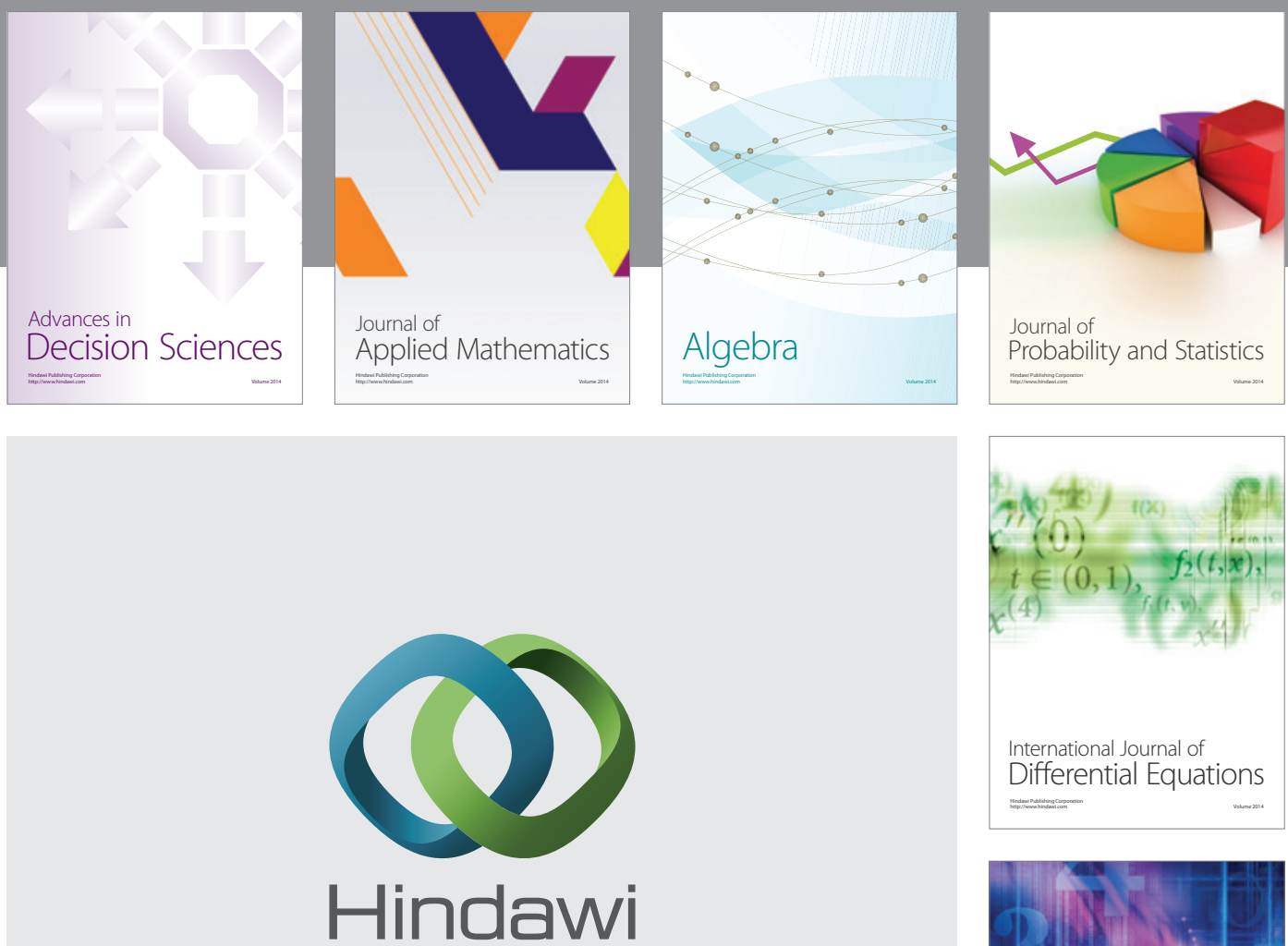

Submit your manuscripts at http://www.hindawi.com
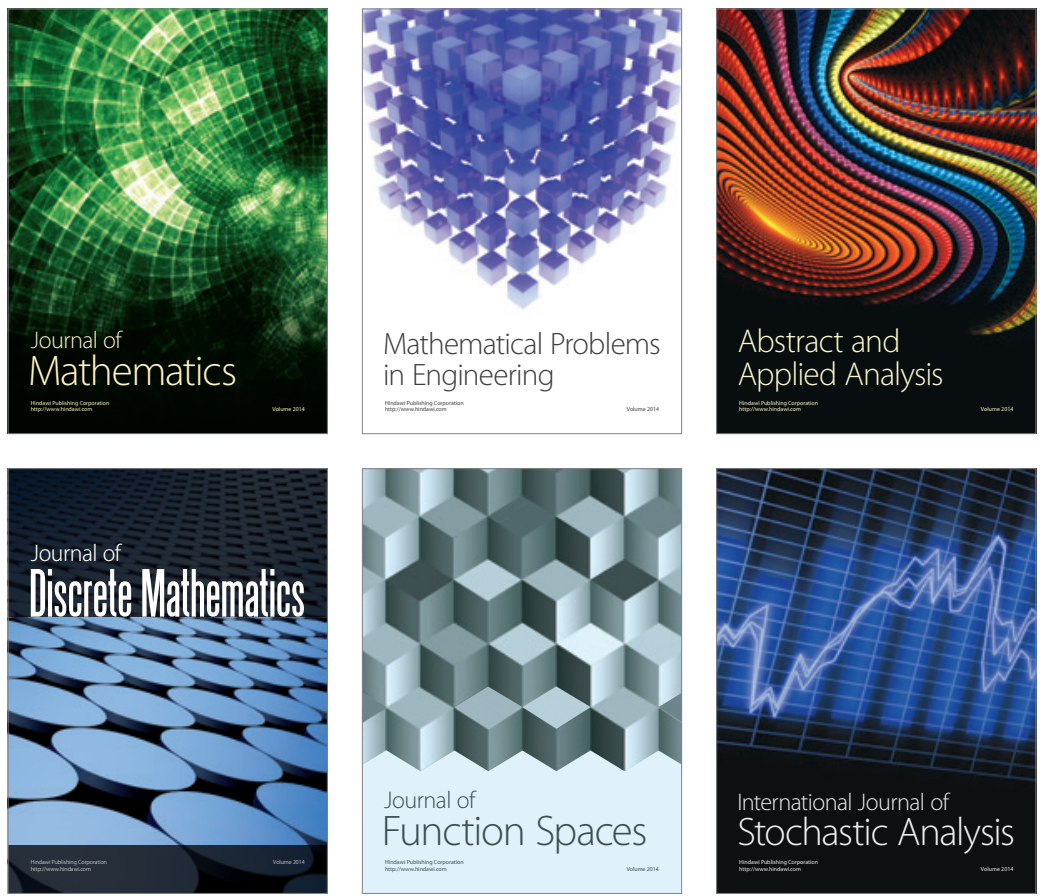

Journal of

Function Spaces

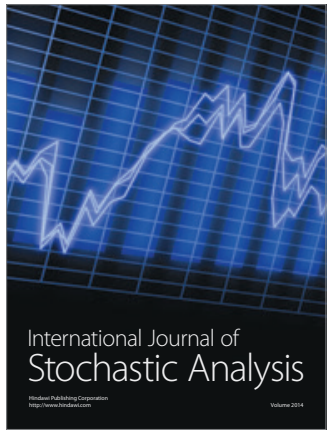

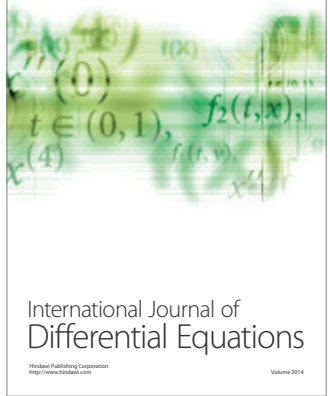
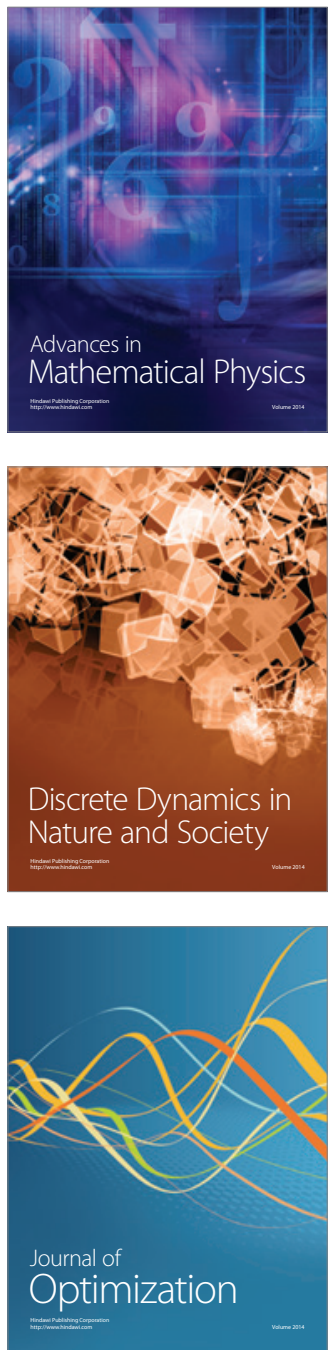